\title{
Epistatic interactions between ancestral genotype and beneficial mutations shape evolvability in Pseudomonas aeruginosa
}

DOI:

10.1111/evo.12958

\section{Document Version}

Accepted author manuscript

Link to publication record in Manchester Research Explorer

Citation for published version (APA):

Gifford, D. R., Toll-Riera, M., \& MacLean, R. C. (2016). Epistatic interactions between ancestral genotype and beneficial mutations shape evolvability in Pseudomonas aeruginosa. Evolution, 70(7), 1659-1666.

https://doi.org/10.1111/evo.12958

\section{Published in:}

Evolution

\section{Citing this paper}

Please note that where the full-text provided on Manchester Research Explorer is the Author Accepted Manuscript or Proof version this may differ from the final Published version. If citing, it is advised that you check and use the publisher's definitive version.

\section{General rights}

Copyright and moral rights for the publications made accessible in the Research Explorer are retained by the authors and/or other copyright owners and it is a condition of accessing publications that users recognise and abide by the legal requirements associated with these rights.

\section{Takedown policy}

If you believe that this document breaches copyright please refer to the University of Manchester's Takedown Procedures [http://man.ac.uk/04Y6Bo] or contact uml.scholarlycommunications@manchester.ac.uk providing relevant details, so we can investigate your claim.

\section{OPEN ACCESS}


1 Epistatic interactions between ancestral genotype and beneficial mutations shape evolvability

4 Danna R. Gifford ${ }^{1,2, *}$ Macarena Toll-Riera ${ }^{1,3}$, and R. Craig MacLean ${ }^{1}$

6 Short title: Constraints on evolvability by magnitude epistasis

$7 \quad$ * Corresponding author: danna.gifford@manchester.ac.uk

8 Telephone: +441612755376

$9 \quad{ }^{1}$ Address

10 Department of Zoology, University of Oxford, South Parks Road, Oxford OX1 3PS, United

11 Kingdom

$12 \quad{ }^{2}$ Present address

13 Faculty of Life Sciences, University of Manchester, Michael Smith Building, Dover St, Manchester

14 M13 9PL United Kingdom

\section{$15 \quad{ }^{3}$ Present address}

16 Institute of Evolutionary Biology and Environmental Sciences, University of Zurich,

17 Winterthurerstrasse 190, CH-8057 Zurich, Switzerland

18

19 Data: Archived in Dryad (http://datadryad.org/) and NCBI Sequence Read Archive (SRA,

20 http://www.ncbi.nlm.nih.gov/sra/) upon manuscript acceptance

21 Keywords: experimental evolution, evolvability, epistasis, antibiotic resistance 


\section{Abstract}

23 The idea that interactions between mutations influence adaptation by driving populations to low and

24 high fitness peaks on adaptive landscapes is deeply ingrained in evolutionary theory. Here we

25 investigate the impact of epistasis on evolvability by challenging populations of two Pseudomonas

26 aeruginosa clones bearing different initial mutations (in rpoB conferring rifampicin resistance, and

27 the type IV pili gene network) to adaptation to a medium containing L-serine as the sole carbon

28 source. Despite being initially indistinguishable in fitness, populations founded by the two ancestral

29 genotypes reached different fitness following 300 generations of evolution. Genome sequencing

30 revealed that the difference could not be explained by acquiring mutations in different targets of

31 selection: the majority of clones from both ancestors converged on one of two strategies, 1)

32 acquiring mutations in either PA2449 (gcsR, an L-serine-metabolism RpoN enhancer binding

33 protein) or 2) protease genes. Additionally, populations from both ancestors converged on loss-of-

34 function mutations in the type IV pili gene network, either due to ancestral or acquired mutations.

35 No compensatory or reversion mutations were observed in RNA polymerase genes, in spite of the

36 large fitness costs typically associated with mutations in $r p o B$. Although current theory points to

37 sign epistasis as the dominant constraint on evolvability, these results suggest that the role of

38 magnitude epistasis in constraining evolvability may be under-appreciated. The contribution of

39 magnitude epistasis is likely to be greatest under the biologically-relevant mutation supply rates that

40 make back-mutations probabilistically unlikely.

\section{Introduction}

Epistatic interactions between novel beneficial mutations and the ancestral genotype they

44 arise in are thought to shape the course of adaptive evolution by restricting the availability of

45 evolutionary trajectories (Phillips et al. 2000; de Visser et al. 2011). As a consequence, ancestral 
47 beneficial mutations, a trait known as 'evolvability'. Differences in evolvability can arise even if

48 selection acts on the same adaptive targets (Colegrave and Collins 2008). A sizable number of

49 studies have shown that ancestral fitness can constrain evolvability (e.g. Burch and Chao 2000;

50 Silander et al. 2007; MacLean et al. 2010; Chou et al. 2011; Gifford et al. 2011; Khan et al 2011;

51 Rokyta et al. 2011; Salverda et al. 2011; Kryazhimskiy et al. 2014; Wong and Seguin 2015), a

52 phenomenon known as diminishing returns epistasis. Diminishing returns epistasis is the tendency

53 for beneficial mutations to confer a smaller benefit in genetic backgrounds of higher fitness (de

54 Visser et al. 2011). These studies match predictions from theoretical models of adaptive evolution

55 predicting that the average fitness of beneficial mutations should be proportional to initial fitness in

56 populations climbing the same adaptive peak (Martin and Lenormand 2006). It is a special case of a

57 more general form of epistasis known as 'magnitude epistasis', defined as the interaction between

58 two mutations that produces a non-additive effect on fitness, without changing the sign (i.e.

59 beneficial/deleterious) of the effect of either mutation (de Visser et al. 2011). Magnitude epistasis is

60 in turn contrasted with sign epistasis, which occurs when the sign of a mutation is conditional on

61 genetic background. Theoretical models predict that sign epistasis will constrain evolvability by

62 restricting populations to certain adaptive peaks (e.g. Weinreich et al. 2005;Kvitek and Sherlock

63 2011; Schenk et al. 2013; Szamecz et al. 2014), whereas magnitude epistasis (including diminishing

64 returns) will restrict the height of adaptive peaks.

Although the roles of diminishing returns epistasis and sign epistasis in evolvability are well-supported by theoretical models and empirical tests, the role of magnitude epistasis, in the

67 general sense, is less concrete. Determining the role of magnitude epistasis in evolvability is

68 important because magnitude interactions are common among pairs of mutations (de Visser et al.

692011 and references therein; Chou et al. 2014). A small, but growing, number of studies that suggest

70 that magnitude epistasis may also influence evolvability (Woods et al. 2011; Kryazhimskiy et al.

71 2014; Szamecz et al. 2014). Evolution experiments using ancestors with similar initial fitness can 
72 demonstrate whether or not magnitude epistasis can constrain evolvability, under conditions that are

73 not solely dictated by diminishing returns, e.g. when ancestral genotypes differ genetically, but not

74 in fitness. This is particularly important in the context of antibiotic resistance, where different

75 resistance mutations may differ in their ability to recover fitness through compensatory mutations,

76 and reversions to sensitivity are rare (Andersson and Hughes 2010; MacLean et al. 2010; Hall et al.

77 2010; Barrick et al. 2010), which may trap populations on local fitness optima.

78 Here we show that evolvability can be constrained by magnitude epistasis that does not

79 result from the diminishing returns-type pattern observed through acquiring multiple beneficial

80 mutations. In our study, we used experimental evolution with two Pseudomonas aeruginosa strains

81 having similar fitness in a simple growth environment (a defined medium with L-serine as the sole

82 carbon source). The strains differed in two protein coding genes: $r p o B$ (SNP versus indel) and pilQ

83 (frame-shift versus wild-type). Mutations in $r p o B$ that confer rifampicin resistance are known to

84 impose large fitness costs through defects in transcription (Qi et al. 2014). Loss of PilQ function

85 leads to the loss of external type IV pili (Martin et al. 1993), which is associated with the loss of

86 twitching motility (Mattick 2002) and decreased virulence (Persat et al. 2015). Despite remarkably

87 parallel evolution at the genetic pathway-level, the two strains differed significantly in the fitness

88 benefit of acquired beneficial mutations. In addition, the genotype initially capable of expressing

89 external pili tended to acquire loss of function mutations in pili genes. No reversions of the $r p o B$

90 resistance alleles were observed, suggesting that the difference of evolvability was not due to a

91 difference in reversability of SNPs versus small indels. Rather, the difference in evolvability was

92 due to epistasis from one or both of the pre-existing mutations.

\section{Methods}

\section{Strains and growth medium}

96 The ancestral strains used in our experiment were isolated from independent populations of a 
97 laboratory strain of $P$. aeruginosa PAO1 that were selected for resistance to a growth-inhibiting

98 dose of rifampicin (64 mg/l) during a one-day fluctuation assay (MacLean and Buckling 2009).

99 Sanger sequencing of $r р о B$ initially revealed that each clone carried a single mutation in the

100 rifampicin binding pocket of $r p o B$ (alleles rpoBS536F and $r p o B+\mathrm{P} 518$, MacLean and Buckling

101 2009). Whole genome re-sequencing of the strains by Illumina HiSeq2000 indicated the presence

102 of these mutations, and revealed one additional mutation in a protein coding gene in the same

103 genetic background as the rpoBS536F mutation: a pilQ frame-shift (deletion of $\mathrm{T}$ at genomic

104 position 5,677,812 producing a frame-shift 15 residues from the $\mathrm{N}$-terminus) and two intergenic

105 mutations (SNPs at genomic positions $1,060,840 \mathrm{G}$ to $\mathrm{A}$, and 5,036,891 A to C). We will identify

106 these ancestral clones by their protein-coding changes: S536F/PilQfs and +P518. Strains were

107 grown in minimal culture medium supplemented with L-serine as the carbon source [M9-serine:

$1083.75 \mathrm{~g} / 1 \mathrm{~L}-$-serine, $10.5 \mathrm{~g} / 1 \mathrm{M} 9$ Broth, and $1 \mathrm{ml}$ of $\left.1 \mathrm{M} \mathrm{MgSO}_{4}\right]$.

\section{Selection experiment}

111 The selection experiment was conducted in conjunction with a previous study (see Gifford and

112 MacLean 2013). Forty populations each were founded from single clones of S536F/PilQfs and

$113+$ P518. Populations were grown statically (i.e. without constant shaking) at $37{ }^{\circ} \mathrm{C}$ in 96 -well

114 microtitre plates. Populations were propagated by serial transfer every $24 \mathrm{~h}$ for 45 transfers, diluting

115 by $1 / 200$ into $200 \mu \mathrm{l}$ fresh M9-serine using a 96-pin replicator that transfers $\sim 1 \mu 1$ of culture. Every

11610 transfers, corresponding to approximately 76 generations, $50 \%$ of each population was stored at $117-80{ }^{\circ} \mathrm{C}$ in $50 \%$ glycerol.

119 Single-mutation fixation experiment

120 To determine the contribution of individual mutations, we isolated individual beneficial mutations

121 using a mixed-marker experiment. 60 populations were initially founded by a 1:1 mix of YFP- 
122 marked and unmarked cells. S536F/PilQfs and +P518 were tagged with a YFP genetic marker

123 (accession no. DQ493879) using the mini-Tn7 insertion protocol (Choi and Schweizer 2006).

124 Environment and transfer regime was as for the 270-generation experiment, except duration was

125 reduced to 14 transfers. The YFP marker was used to detect selective sweeps. The proportion of

126 YFP-marked cells was measured every second day from a $10 \mu 1$ population sample, counting on

127 average $10^{3}$ cells by cytometry (BD Accuri'TM $\mathrm{C} 6$ with Csampler ${ }^{\mathrm{TM}}$, BD Biosciences). When the

128 YFP-marker was fixed $(>90 \%)$ or lost $(<10 \%)$ from a population, we stored $50 \%$ of that population

129 at $-80{ }^{\circ} \mathrm{C}$ in $50 \%$ glycerol. When populations were revived for sequencing and fitness assays, we

130 checked that we recovered the correct marker.

132 Competitive fitness assays

133 Competitive fitnesses was assayed using flow cytometry. Evolved strains were competed against

134 their ancestor, scaled by the initial fitness deficit of the ancestor relative to P. aeruginosa PAO1. To

135 revive the frozen evolved and ancestral strains, we first pin replicated $1 \mu$ of the frozen population 136 into $200 \mu \mathrm{l} \mathrm{M9-serine.} \mathrm{Strains} \mathrm{were} \mathrm{grown} \mathrm{in} \mathrm{M9-serine} \mathrm{for} 48 \mathrm{~h}$ prior to competition experiments 137 to physiologically acclimate to the medium (first for a $24 \mathrm{~h}$ growth period, then a 1 in 200 dilution, 138 followed by a second $24 \mathrm{~h}$ growth period). We created mixtures of YFP-marked and unmarked cells 139 and quantified fitness according to the change in frequency across a 24-hour growth period. The 140 formula for the fitness of strain A relative to strain B over a fixed time period ( $24 \mathrm{~h}$, approximately 1417.64 generations $)$ is $w_{\mathrm{AB}}=\log _{2}\left(D A_{f} / A_{\mathrm{i}}\right) / \log _{2}\left(D B_{f} / B_{i}\right)$, where subscripts $i$ and $f$ indicate initial and 142 final frequencies, respectively, and $D$ is the dilution factor used to initiate the competition 143 experiment $(1 / 200)$. Changes in fitness are expressed as selection coefficients $(s)$ relative to 144 ancestral fitness, $s=w_{\text {evolved }} / w_{\text {ancestor }}-1$ (Lenski et al. 1991). Data were analyzed using R 3.0.1 (R 145 Core Team 2013), and are available as online supplemental material. 


\section{Genomic DNA extraction and sequencing pipeline}

148 We analyzed 22 S536F/PilQfs-derived and $18+$ P518-derived lineages from the 270 generation

149 selection experiment, and 18 S536F/PilQfs-derived $22+$ P518-derived lineages from the single-

150 mutation fixation experiment. Genomic DNA (gDNA) was extracted with the Promega Wizard

151 Genomic DNA Purification Kit (Promega, UK). Complete details on the extraction method are

152 available in the online supplementary methods. Sequencing was performed using Illumina

153 HiSeq2000 with 100 bp paired-end reads, at the Wellcome Trust Centre for Human Genetics

154 (Oxford, UK). Reads were filtered and analysed for mutations according to the pipeline described in 155 San Millan et al. 2014 (full details also available in the online supplementary material). After 156 filtering, median coverage depth for each clone ranged from $35 \mathrm{X}$ to $80 \mathrm{X}$. Sequences are deposited 157 in the NCBI Sequence Read Archive (SRP053291).

159 Results and discussion

160 Evolutionary trajectories

The ancestral strains used in our experiment were two clones isolated from a strain of $P$. aeruginosa $\mathrm{PAO}$, bearing different $r p o B$ and pilQ alleles: S536F/PilQfs and +P518 (see methods and MacLean and Buckling, 2009). These clones had similar deficits in fitness (w) relative to their common PAO1 ancestor, and were chosen in an effort to remove fitness as a confounding effect on evolvability (S536F/PilQfs $w=0.82 \pm 0.054$ SE vs. + P5 $18 w=0.784 \pm 0.031$ SE, two-sample $t$-test: $\left.t_{10}=1.42, p=0.18\right)$.

We adapted 40 independent populations from each genetic background to growth on M9

168 salts with L-serine as the sole carbon source. Following 270 generations of adaptation,

169 S536F/PilQfs lineages had higher fitness than +P518 lineages (S536F/PilQfs $w=1.344 \pm 0.074$ SE, $170+\mathrm{P} 518 w=1.044 \pm 0.065 \mathrm{SE}$, two-sample $t$-test: $t_{17.9}=-2.58, p=0.02$; fitness values scaled to initial 
171 fitness deficit of ancestors). In both genetic backgrounds, fitness measured against the ancestor

172 increased monotonically with the number of generations of evolution, until approximately

173 generation 150. Clones that had evolved for more than 150 generations did not have higher fitness

174 against the ancestor than those from generation 150 suggesting that either populations are close to 175 reaching a local fitness optimum, or that mutations provide non-transitive fitness benefits (Figure

176 1).

177 Genetic basis of adaptation

We examined the underlying genetic basis for adaptation by whole-genome sequencing 40 independently evolved clones (22 of S536F/PilQfs and 18 of +P518) from the end of the experiment. Clones isolated from lineages descendant from both ancestors had between one and four mutations; although +P518-descendant clones had slightly more mutations on average, the difference was not significant $(2.09 \pm 0.15 \mathrm{SE}$ vs. $2.33 \pm 0.18 \mathrm{SE}$; Negative binomial GLM: intercept $z=5.0, p<0.001$; genotype $z=0.514, p=0.61)$. This indicates that the difference in evolvability did not arise from a difference in the number of mutations fixed.

The genetic bases of adaptation in descendants of both ancestors was remarkably similar

(Figure 2 and Tables S1 and S2), but the fitness associated with mutations was strongly contingent on ancestry. Nearly all evolved lineages acquired mutations in one of two selective targets: nonsynonymous SNPs in PA2449, a TyrR-like RpoN enhancer binding protein (Lundgren et al. 2013, recently named $\operatorname{gcs} R$ for glycine cleavage system regulator, Sarwar et al. 2016), and small indels leading to putative loss-of-function mutations in two protease complexes that share similar protein targets and functions ( $c l p A$ from the ClpAP serine protease complex, and $f t s H, h f l C$, and $h f l K$ from

192 the FtsH/HflKC complex, Lundgren et al. 2013; Zhou and Jin 1998). Mutations in pili formation 193 genes, most often causing a loss of protein function, were a third target of selection in + P518

194 lineages, but not in S536F/PilQfs lineages, which already had a pili loss of function mutation 195 (discussed below). We also observed four mutations in $\operatorname{cbr} A B$ (involved in amino acid metabolism, 
196 Nishijyo et al. 2001), suggesting it may represent a fourth target of selection. We found no evidence

197 for large indels, IS transposition, or genome rearrangements.

The frequency of lineages with a mutation in either PA2449 or proteases (but not both) was

199 equal in both genetic backgrounds (Fisher's exact test: $p=0.73$ ). However, the fitness advantage

200 associated with mutations in these targets was greater in S536F/PilQfs lineages than +P518 lineages

201 (Figure 3; Type III ANOVA: ancestor genotype $F_{1,29}=21.3, p<0.001$, selective target $F_{1,29}=12.0$,

$202 p=0.002$, interaction $F_{1,29}=4.75, p=0.038$ ), although overlap at the level of individual mutations was

203 low (addressed below). The difference in selection coefficient was more pronounced for PA2449

204 mutations (two-sample t-test: $t_{16.21}=3.88, p=0.001$ ) than protease mutations (two-sample $t$-test:

$\left.205 \mathrm{t}_{11.97}=1.97, p=0.07\right)$. This suggests that epistasis between the ancestral genotype and novel

206 beneficial mutations, but not the accessibility of those beneficial mutations, determined the

207 evolvability of the two ancestral genotypes.

208 Although the genetics of adaptation were highly similar in S536F/PilQfs and +P518

209 lineages, some differences are apparent. The first notable difference is that five S536F/PilQfs

210 descendant lineages had mutations in both PA2449 and a protease gene, but the fitness of these five

211 double mutants was indistinguishable from the 10 lineages with PA2449 mutations but no protease

212 mutations (Wilcoxon rank-sum test, $W=37, p=0.16$, Figure 3), indicating that PA2449/protease

213 double mutants were not responsible for S536F/PilQfs's greater evolvability. The second difference

214 was the acquisition of mutations type IV pili formation genes (pil genes, tsaP and algR) in 15 of 18

215 lineages in the +P518 background only. This difference likely arises due to the ancestral PilQfs

216 mutation in S536F/PilQfs, as mutations in pilQ or any of the genes mutated in evolved +P518

217 clones should result in a non-piliated phenotype (Mattick 2002; Ayers et al. 2009; Leighton et al.

218 2015; Hmelo et al. 2015). The convergence of both ancestral genotypes on pili gene defects

219 suggests that a non-piliated phenotype confers some benefit on its own. Notably, however, all but

220 one of the +P518 lineages carrying a PA2449 mutation also carried a pili gene mutation, suggesting 
221 that the larger fitness benefit of PA2449 mutations in S536/PilQfs lineages over +P518 lineages

222 does not arise due to the absence of pili generally. Third, although the same genes were mutated in

223 both backgrounds, there was incomplete overlap in the specific mutations acquired in the genes

224 targeted by selection. An alternate explanation for our results is that S536F/PilQfs by chance

225 acquired larger-effect mutations than + P518. An approach to determine whether this is the case

226 would be to use allelic exchange to place the acquired mutations into both ancestral backgrounds

227 (Hmelo et al. 2015).

An assumption of this analysis is that 'accessory mutations' co-occurring with the primary

229 adaptive mutations had negligible affects on fitness. To test this assumption, we sequenced 40

230 individual clones selected for single beneficial mutations in a mixed-marker experiment (see

231 Methods; Table S3). For the 13 clones found to have only a PA2449 mutation, the fitness advantage

232 equaled that of strains with both a PA2449 mutation and another mutation (Figure 3, Type III

233 ANOVA: ancestor genotype $F_{1,26}=12.7, p=0.001$; presence of accessory mutation $F_{1,26}=1.51$,

$234 p=0.23$, insignificant interaction term dropped). This suggests that PA2449 contributed the bulk of

235 the fitness benefit and accessory mutations were of little consequence for evolvability, but we note

236 that we had low power to detect small differences in the case of + P518 lineages. If we further

237 restrict the analysis to evolved clones that possessed only a PA2449 mutation and a pili gene

238 mutation ( $n=3$ from each ancestral background), the difference in selection coefficient between

239 S536F/PilQfs and +P518 remains significant (S536F/PilQfs $s=1.08 \pm 0.038$ SE vs. + P518

$240 s=0.242 \pm 0.112$ SE; two-sample $t$-test: $\left.t_{2.42}=6.84, p=0.01\right)$.

\section{Environment-specific roles of the targets of selection}

242 Of the targets of selection, PA2449 ( $g s c R$, Sarwar et al. 2016) has the most obvious role in L-serine

243 metabolism. PA2449 is an RpoN enhancer binding protein that initiates RpoN-controlled

244 transcription of genes for L-serine and L-glycine metabolism via regulation of the glycine cleavage

245 system (Lundgren et al. 2013, Sarwar et al. 2016). The SNPs we observed in PA2449 are likely to 
246 improve its function when L-serine is a carbon source, rather than cause loss of function, as PA2449

247 knockouts have severely limited growth on L-serine (Lundgren et al. 2013). PA2449 mutations are

248 also not likely to compensate for the defect in RpoB. PA2449 mutations are only beneficial on L-

249 serine and - to a lesser extent - L-glycine, which is part of the same metabolic pathway as L-serine

250 (Lundgren et al. 2013, Sarwar et al. 2016). The same mutations are neutral or deleterious on other

251 carbon sources (e.g. glucose and KB, see online supplemental methods and Figure S1). Why

252 S536F/PilQfs should gain a bigger benefit than +P518 from PA2449 mutations is unclear. In E. coli,

253 RNA polymerase (RNAP) with the S531F form of RpoB (equivalent to S536F in P. aeruginosa)

254 behaves like 'stringent' RNAP, shifting gene expression toward genes regulated by the alternative

255 sigma factors (Zhou and Jin 1998). S536F also has wild-type levels of transcriptional efficiency,

256 but altered gene expression, relative to other rpoB alleles (Qi et al. 2014). However, Hall et al. 2010

257 found that + P518 had low evolvability among a set of rpoB mutants adapting to a complex

258 environment, but did not determine the basis for the difference in evolvability (i.e. number of

259 mutations or epistasis). Together, the data suggest that the +P518 mutation may have many

260 pleiotropic effects on fitness. Given that PA2449 is a transcriptional activator, it is likely that the

261 differences in evolvability stem from the mutations in $r p o B$, which is the master regulator of

262 transcription. The convergence of + P518 and S536F/PilQfs lineages on a pili-less phenotype is

263 indirect evidence that the $r p o B$ mutations are responsible for the observed magnitude epistasis.

264 However, we cannot strictly eliminate the possibility that the loss of PilQ in S536F/PilQfs clone

265 specifically affects the fitness effect of PA2449 mutations, although we note that a +P518 lineage

266 with both a SNP in PA2449 and a premature stop in PilQ did not have significantly higher fitness

$267(w=0.948 \pm 0.0914 \mathrm{SE})$. Interestingly, mutations in DNA topoisomerase, which is also involved in

268 bacterial gene expression, have also been shown to affect evolvability (Woods et al. 2011). It

269 remains to be determined whether epistatic interactions occur more in core regulators of gene

270 transcription. 
In contrast, what benefit the loss of proteases and pili may provide in this environment is

272 unclear. We observed loss-of-function mutations in four protease components from two complexes:

$273 \operatorname{clp} A$ (from ClpAP), and $f t s H, h f l C$, and $h f l K$ (from FtsH/HflKC), and several pili genes. The

274 protease complexes are involved both in general proteolysis and disaggregation of mis-folded

275 proteins, and specific regulation of transcription factors for fine-tuning cellular metabolism

276 (Dougan 2013; Paget 2014). ClpA has been shown to regulate some of the same genes as PA2449 in

277 P. aeruginosa PAO1, including genes involved in quorum-sensing (e.g. pyocyanin, elastase, and

278 C4-homoserine lactone, Diggle et al. 2002). This suggests that PA2449 and proteases may both

279 affect the expression of parts of the metabolic pathway involved in L-serine metabolism. One

280 possibility is via RpoN, as PA2449 is an activator of RpoN-controlled transcription, and ClpA and

281 RpoN are in the same protein-protein interaction network in Escherichia coli, (Rajagopala et al.

282 2014). Loss of pili is likely an adaptation to growth in a non-shaken environment, as the absence of

283 pili is associated with an increase in swimming speed in P. aeruginosa PAO1 (Taylor and Buckling

284 2010). Alternatively, the loss of pili production may also reduce demand for RpoN, as pilus

285 production is under RpoN control (Ishimoto and Lory 1989). Several of the accessory genes

286 mutated are also transcriptional regulators or are involved in RpoN-controlled transcription (Table

287 S2), suggesting that increasing RpoN availability may nominally increase fitness.

Among our populations, we found no evidence for reversion of resistance through back

mutations in $r p o B$, nor any evidence for compensatory mutations through second-site mutations in

any RNA polymerase genes. The absence of reversion is consistent with previous evolution studies

291 with rifampicin resistant populations in antibiotic-free environments (e.g. Hall et al. 2010).

292 However, the absence of any putative compensatory mutations in $r p o A, r p o B$ or $r p o C$ contrasts with

293 previous studies (Reynolds 2000; Hall et al. 2011; Comas et al. 2012), suggesting improving L-

294 serine metabolism confers larger fitness benefits than repairing RNA transcription. Previous results

295 found that selecting rifampicin-sensitive PAO1 on L-serine also results in PA2449, clpA, and pili 
gene mutations (Gifford et al. 2015), strongly suggesting that the observed mutations reflect general

297

298

299

300

301

302

303

304

305

306

307 308

309

310

311

312

313 adaptation to the culture medium or laboratory conditions, and not compensatory adaptation to recover the cost of rifampicin resistance. The absence of reversions and compensatory mutations is surprising, given the large initial cost of rifampicin resistance born by both mutants. The ability to adapt to or compensate for the costs of antibiotic resistance is thought to influence which specific resistance mutations are maintained at the population level over evolutionary time scales (Andersson 2006), but we were not able to test this prediction here. This highlights the importance of considering mutational supply in predicting adaptive trajectories; as PA2449 mutations are beneficial in the rifampicin-sensitive PAO1 background (Gifford et al. 2015), reversion should still confer a benefit. Although reversion is a valid mutational step that would allow further adaptation, the probability of the mutations is low enough that the lineages were effectively trapped in the region of a fitness peak by epistasis, and by the availability (or lack thereof) of reversion mutations.

\section{Conclusions}

Our results suggest that magnitude epistasis can constrain evolvability at a practical level, in agreement with previous studies (Woods et al. 2011; Kryazhimskiy et al. 2014; Szamecz et al. 2014). However, further work is needed to determine which genes may constrain magnitude epistasis at a global level. Our results are particularly relevant to the recovery of fitness costs associated with antibiotic resistance. Chromosomal antibiotic resistance mutations often occur in genes involved in gene expression and protein synthesis, and thus are likely to interact with many other genes on a global level (e.g. rpoB for rifampicin, gyrA for fluoroquinolones, $r p s L$ for streptomycin, fusA for fusidic acid, Andersson and Hughes 2010). If resistance mutations have a tendency to interact epistatically with generally beneficial mutations, this could have important consequences for the long-term maintenance of resistance in antibiotic-free environments. Previous studies of compensation in antibiotic resistant lineages have focused on the short-term 
consequences of fitness costs. Our results are the first to show that resistance can also have long-

322 term consequences for evolvability, which could have important implications in the migration and

323 subsequent adaptation of resistant strains between agricultural and clinical hosts.

\section{Acknowledgements}

326 We thank Mila Kojadinovic for assistance in gDNA extractions. The research leading to these 327 results has received funding from the European Research Council under the European Union's 328 Seventh Framework Programme (FP7/2007-2013)/ ERC grant agreement no. 281591, and from The 329 Royal Society to RCM. DRG was funded by the National Sciences and Engineering Research 330 Council (NSERC, Canada), the Clarendon Fund and Somerville College. We thank the High331 Throughput Genomics Group at the Wellcome Trust Centre for Human Genetics (funded by 332 Wellcome Trust grant reference 090532/Z/09/Z and MRC Hub grant G090074791070) for the 333 generation of the sequencing data.

\section{Authors' contributions}

336 Conceived of the experiment: DRG and RCM. Conducted laboratory work: DRG. Wrote and 337 executed mutation calling pipeline: MT-R. Analysed data and wrote the manuscript: all authors.

\section{Conflicts of interest}

340 The authors declare no conflicts of interest.

\section{References}

343 Andersson DI, Hughes D. 2010. Antibiotic resistance and its cost: is it possible to reverse 344 resistance? Nature Reviews Microbiology 8:260-271.

345 Andersson DI. 2006. The biological cost of mutational antibiotic resistance: any practical 
conclusions? Current Opinion in Microbiology 9:461-465.

347

Ayers, M, Sampaleanu, L, Tammam, S, Koo, J, Harvey, H, Howell, P, and Burrows, L. 2009. PilM/N/O/P proteins form an inner membrane complex that affects the stability of the Pseudomonas aeruginosa type IV pilus secretin. Journal of molecular biology, 394(1):128142.

Bailey, SF, Rodrigue, N, and Kassen, R. 2015. The effect of selection environment on the probability of parallel evolution. Molecular Biology and Evolution (online early).

Barrick, JE, Kauth, MR, Strelioff, CC, \& Lenski, RE. 2010. Escherichia coli rpoB mutants have increased evolvability in proportion to their fitness defects. Molecular Biology and Evolution, 27: $1338-1347$.

Choi KH, Schweizer HP. 2006. mini-Tn7 insertion in bacteria with single att $\operatorname{Tn} 7$ sites: example Pseudomonas aeruginosa. Nature Protocols 1:153-161.

Chou HH, Chiu HC, Delaney NF, Segrè D, Marx CJ. 2011. Diminishing returns epistasis among beneficial mutations decelerates adaptation. Science 332:1190-1192.

Chou, HH, Delaney, NF, Draghi, JA, Marx, CJ. 2014. Mapping the fitness landscape of gene expression uncovers the cause of antagonism and sign epistasis between adaptive mutations. PLoS Genetics, 10(2), e1004149.

Colegrave N, Collins S. 2008. Experimental evolution: experimental evolution and evolvability. Heredity 100:464-470.

Comas I, Borrell S, Roetzer A, Rose G, Malla B, Kato-Maeda M, Galagan J, Niemann S, Gagneux S. 2012. Whole-genome sequencing of rifampicin-resistant Mycobacterium tuberculosis strains identifies compensatory mutations in RNA polymerase genes. Nature Genetics 44:106110.

Costanzo M, Baryshnikova A, Bellay J, Kim Y, Spear ED, Sevier CS, Ding H, Koh JL, Toufighi K, 
371

de Visser JAGM, Hoekstra RF, Van den Ende H. 1997. An experimental test for synergistic epistasis and its application in Chlamydomonas. Genetics 145:815-819.

de Visser JAGM, Hoekstra RF, Van den Ende H. 1997. Test of interaction between genetic markers that affect fitness in Aspergillus niger. Evolution 51:1499-1505.

de Visser JAGM, Cooper, TF, Elena SF. 2011. The cause of epistasis. Proceedings of the Royal Society B 278:3617-3624.

Diggle SP, Winzer K, Lazdunski A, Williams P, Cámara M. 2002. Advancing the quorum in Pseudomonas aeruginosa: MvaT and the regulation of $\mathrm{N}$-acylhomoserine lactone production and virulence gene expression. Journal of bacteriology 184:2576-2586.

Dougan DA. 2013. Regulated Proteolysis in Microorganisms. volume 66. Springer.

Franke, J, Klözer, A, de Visser, JAGM, and Krug, J. 2011. Evolutionary accessibility of mutational pathways. PLoS Computational Biology, 7(8):e1002134.

Gifford DR, MacLean RC. 2013. Evolutionary reversals of antibiotic resistance in experimental -populations of Pseudomonas aeruginosa. Evolution 67:2973-2981.

Gifford DR, Schoustra SE, Kassen R. 2011. The length of adaptive walks is insensitive to starting fitness in Aspergillus nidulans. Evolution 65:3070-3078.

Gifford DR, Toll-Riera M, Kojadinovic M, MacLean RC. 2015. Here's to the losers. The American Naturalist, 186(1):41-49.

Hall AR, Griffiths VF, MacLean RC, Colegrave N. 2010. Mutational neighbourhood and mutation supply rate constrain adaptation in Pseudomonas aeruginosa. Proceedings of the Royal Society B 277:643-650.

Hall AR, Iles JC, MacLean RC. 2011. The fitness cost of rifampicin resistance in Pseudomonas 

aeruginosa depends on demand for RNA polymerase. Genetics 187:817-822.

394 Hmelo LR, Borlee BR, Almblad H, Love ME, Randall TE, Tseng BS, Lin C, Irie Y, Storek KM, 395 Yang JJ, Siehnel RJ, Howell PL, Sing, PK, Tolker-Nielsen TT, Parsek MR, Schweizer HP, Harrison JJ. 2015. Precision-engineering the Pseudomonas aeruginosa genome with two-step

Khan AI, Dinh DM, Schneider D, Lenski RE, Cooper TF. 2011. Negative epistasis between allelic exchange. Nature Protocols 10(11):1820-1841.

Ishimoto KS, Lory S. 1989. Formation of pilin in Pseudomonas aeruginosa requires the alternative sigma factor (RpoN) of RNA polymerase. Proceedings of the National Academy of Sciences 86:1954-1957.

403

Kryazhimskiy, S, Dushoff, J, Bazykin, GA, and Plotkin, JB. 2011. Prevalence of epistasis in the evolution of influenza A surface proteins. PLoS Genetics, 7(2):e1001301.

Kryazhimskiy S, Rice DP, Jerison E, Desai MM. 2014. Global epistasis makes adaptation predictable despite sequence-level stochasticity. Science 344:1519-1522.

Kvitek DJ, Sherlock G. 2011. Reciprocal sign epistasis between frequently experimentally evolved 408 adaptive mutations causes a rugged fitness landscape. PLoS Genetics 7:e1002056.

Lalić J, Elena SF. 2012. Magnitude and sign epistasis among deleterious mutations in a positivesense plant RNA virus. Heredity 109:71-77.

411 Leighton, TL, Buensuceso, R, Howell, PL, and Burrows, LL. (2015). Biogenesis of Pseudomonas aeruginosa type IV pili and regulation of their function. Environmental Microbiology (online

414 Lenski, RE, Rose, MR, Simpson, SC, and Tadler, SC. 1991. Long-term experimental evolution in Escherichia coli. I. Adaptation and divergence during 2,000 generations. The American 
417 Lundgren B, Thornton W, Dornan M, Villegas-Peñaranda L, Boddy C, Nomura C. 2013. The gene 418 PA2449 is essential for glycine metabolism and pyocyanin biosynthesis in Pseudomonas 419 aeruginosa PAO1. Journal of Bacteriology 195:2087-2100.

420 Lunzer M, Golding G, Dean A. 2010. Pervasive cryptic epistasis in molecular evolution. PLoS Genetics 6:e1001162.

422 MacLean R, Hall A, Perron G, Buckling A. 2010. The population genetics of antibiotic resistance: 423 integrating molecular mechanisms and treatment contexts. Nature Reviews Genetics 11:405414.

425 MacLean RC, Buckling A. 2009. The distribution of fitness effects of beneficial mutations in Pseudomonas aeruginosa. PLoS Genetics 5:e1000406, doi:10.1371/journal.pgen.1000406.

Martin PR, Hobbs M, Free PD, Jeske Y, \& Mattick JS. 1993. Characterization of pilQ, a new gene required for the biogenesis of type 4 fimbriae in Pseudomonas aeruginosa. Molecular Microbiology 9(4):857-868.

Martin G and Lenormand T. 2006. A general multivariate extension of Fisher's geometrical model and the distribution of fitness effects across species. Evolution, 60(5):893-907.

432 Mattick, JS. 2002. Type IV pili and twitching motility. Annual Reviews in Microbiology, 56(1):289314.

434 Nishijyo T, Haas D, Itoh Y. 2001. The CbrA-CbrB two-component regulatory system controls the 435 utilization of multiple carbon and nitrogen sources in Pseudomonas aeruginosa. Molecular 436 microbiology 40:917-931.

437 Paget M. 2014. Prokaryotic Gene Regulation by Sigma Factors and RNA Polymerase. In: Molecular Life Sciences. Springer. 
439 Persat A, Inclan YF, Engel JN, Stone HA, and Gitai Z. 2015. Type IV pili mechanochemically regulate virulence factors in Pseudomonas aeruginosa. Proceedings of the National Academy

442 Poelwijk, FJ, Kiviet, DJ, Weinreich, DM, and Tans, SJ. 2007. Empirical fitness landscapes reveal accessible evolutionary paths. Nature, 445(7126):383-386.

444 Phillips PC, Otto SP, Whitlock MC. 2000. Beyond the average: the evolutionary importance of gene of Sciences, 112(24):7563-7568.

Qi Q, Preston GM, MacLean RC. 2014. Linking System-Wide Impacts of RNA Polymerase Mutations to the Fitness Cost of Rifampin Resistance in Pseudomonas aeruginosa. mBio 5:e01562-14.

Rajagopala SV, Sikorski P, Kumar A, Mosca R, Vlasblom J, Arnold R, Franca-Koh J, Pakala SB, 2014. The binary protein-protein interaction landscape of Escherichia coli. Nature Biotechnology 32:285-290.

Reynolds MG. 2000. Compensatory evolution in rifampin-resistant Escherichia coli. Genetics

Rokyta DR, Joyce P, Caudle SB, Miller C, Beisel CJ, Wichman HA. 2011. Epistasis between $156: 1471-1481$

Romero PA, Arnold FH. 2009. Exploring protein fitness landscapes by directed evolution. Nature beneficial mutations and the phenotype-to-fitness map for a ssDNA virus. PLoS Genetics $7: \mathrm{e} 1002075$ Reviews Molecular Cell Biology 10(12):866-76.

1 R Core Team. 2013. R: a language and environment for statistical computing. R Foundation for 
463

464

465

466

467

468

469

470

471

472

473

474

475

476

477

478

479

480

481

482

San Millan A, Peña-Miller R, Toll-Riera M, Halbert Z, McLean A, Cooper B, MacLean R. 2014. Positive selection and compensatory adaptation interact to stabilize non-transmissible plasmids. Nature Communications 5.

Sarwar Z, Lundgren BR, Grassa MT, Wang MX, Gribble M, Moffat JF, Nomura CT. 2016. GcsR, a TyrR-like enhancer-binding protein, regulates expression of the glycine cleavage system in Pseudomonas aeruginosa PAO1. mSphere 1(2):e00020-16.

Segre D, DeLuna A, Church GM, Kishony R. 2004. Modular epistasis in yeast metabolism. Nature Genetics 37:77-83.

Schenk MF, Szendro IG, Salverda ML, Krug J, and de Visser JAGM. 2013. Patterns of epistasis between beneficial mutations in an antibiotic resistance gene. Molecular Biology and Evolution, 30(8):1779-1787.

Szamecz B, Boross G, Kalapis D, Kovács K, Fekete G, Farkas Z, Lázár V, Hrtyan M, Kemmeren P, Groot Koerkamp MJA, Rutkai E, Holstege FCP, Papp B and Pál C. (2014). The genomic landscape of compensatory evolution. PLoS Biology, 12(8):e1001935-e1001935.

Taylor, TB and Buckling, A. 2010. Competition and dispersal in Pseudomonas aeruginosa. The American Naturalist, 176(1):83-89.

Taylor, TB and Buckling, A. 2011. Selection experiments reveal trade-offs between swimming and twitching motilities in Pseudomonas aerugionsa. Evolution, 65(11):3060-3069.

Weinreich D, Watson R, Chao L. 2005. Perspective: sign epistasis and genetic constraint on evolutionary trajectories. Evolution 59:1165-1174.

483 Weinreich, D, Delaney, N, DePristo, M, and Hartl, D. 2006. Darwinian evolution can follow only 

very few mutational paths to fitter proteins. Science, 312(5770):111-114.

485 Whitlock MC, Phillips PC, Moore FBG, Tonsor SJ. 1995. Multiple fitness peaks and epistasis. 486 Annual Review of Ecology and Systematics :601-629.

487 Wong, A, Seguin K. 2015. Effects of genotype on rates of substitution during experimental 488 evolution. Evolution (Accepted Article, accessed 14/07/2015). doi: 10.1111/evo.12700.

489 Woods RJ, Barrick JE, Cooper TF, Shrestha U, Kauth MR, Lenski RE. 2011. Second-order selection 490 for evolvability in a large Escherichia coli population. Science 331:1433-1436.

491 Zhou YN, Jin DJ. 1998. The rpoB mutants destabilizing initiation complexes at stringently 492 controlled promoters behave like "stringent" RNA polymerases in Escherichia coli. 493 Proceedings of the National Academy of Science of the United Statesof America 95:29084942913. 


\section{Figures and tables}

497 Figure 1: Average fitness $( \pm \mathrm{SE})$ of $P$. aeruginosa lineages selected on L-serine (40 total populations, 498 averaged over subsets of $n=10$ separate populations at each time point, normalized by initial fitness 499 cost associated with rpoB mutations).

501 Figure 2: Mutations in evolved clones. Columns indicate individual clones and rows indicate genes 502 that were mutated. Filled boxes indicate that a mutation was found in that gene (multiple filled 503 boxes in a single column indicate the presence of mutations in multiple genes in the same lineage).

504 Striped boxes indicate the presence of a mutation in the ancestral clone. Where indicated, a number 505 indicates the presence of more than one mutation. Mutated protease genes include $c l p A, f t s H, h f l C$ 506 and $h f l K$. Mutated pili-related genes include pilB, pilC, pilE, pill, pilM, pilQ, pilR, pilW, pilY1, tasP, 507 and $\operatorname{algR}$ (Mattick 2002; Ayers et al. 2009; Leighton et al. 2015). See Table S1 for specific 508 mutations found in each gene.

510 Figure 3: Average fitness $( \pm \mathrm{SE})$ of $P$. aeruginosa clones bearing mutations in either PA2449, 511 protease gene(s), both PA2449 and a protease gene, or neither. Fitness was normalized by initial 512 fitness cost of ancestor strain relative to PAO1. Circles denote clones from the 270 generation 513 selection experiment (also bearing one or more accessory mutations), and squares denote clones 514 from the single-mutation fixation experiment bearing only a single mutation in PA2449 (See Table 515 S1-S3 for a list of all genes mutated in all strains). 


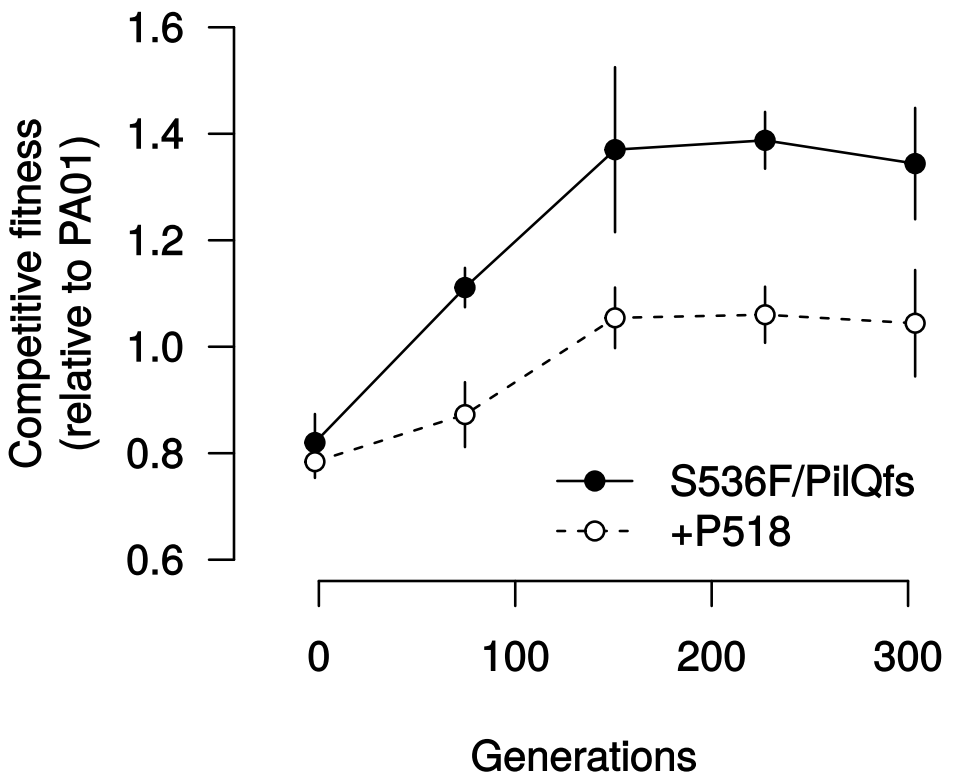




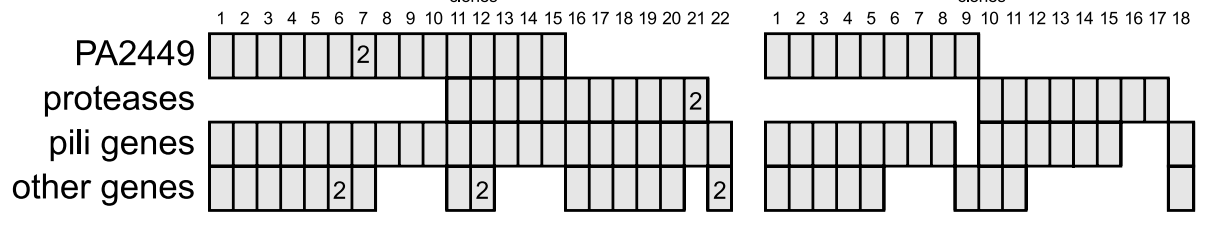




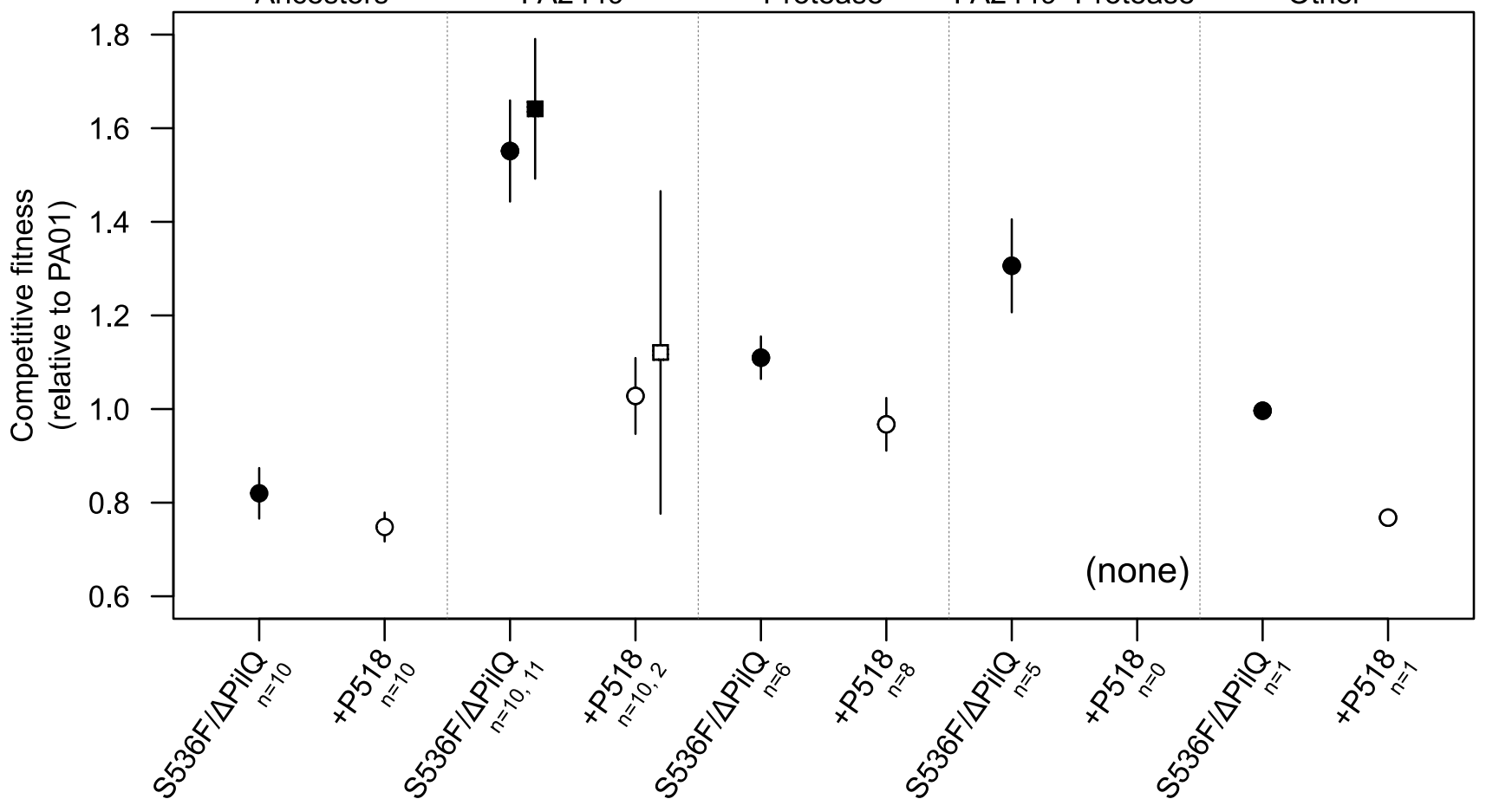




\section{Epistatic interactions between ancestral genotype and beneficial mutations shape evolvability in Pseudomonas aeruginosa}

Danna R. Gifford ${ }^{1,2, *}$ Macarena Toll-Riera ${ }^{1,3}$, and R. Craig MacLean ${ }^{1}$

\section{Supplementary methods}

\section{Genomic DNA extraction and sequencing pipeline}

Whole genome sequencing analysis was performed as described in San Millan et al. (2014). Whole genome sequencing services were provided by the Wellcome Trust Centre for Human Genetics (Oxford, UK) using the Illumina HiSeq 2000 platform with 100 bp paired-end reads. Initial read filtering was done using NIH QC Toolkit (Patel and Jain, 2012). 5' or 3' ends were trimmed if the Phred quality score was less than 20 . Reads were discarded if they were shorter than $50 \mathrm{bp}$ after trimming, if more than $2 \%$ of bases were ambiguous, or if more than $20 \%$ of bases had a Phred score $<$ 20. BWA was used to map filtered reads were mapped to the P. aeruginosa PA01 reference genome (NC_002516.2). Mapped reads were processed to increase the quality of the variant calling: 1) reads with multiple best hits were discarded; 2) duplicated reads were discarded using MarkDuplicates from the Picard package (http://picard.sourceforge.net); 3) reads around indels were locally realigned using RealignerTargetCreator and IndelRealigner from the GATK package to correct for misalignment; and 4) mate pairs were sorted using FixMateInformation in the Picard package. Variant calling was performed with GATK's Unified Genotyper (De Pristo et al., 2011) and Samtools's mpileup (Li et al., 2009). VCFtools (vcf-annotate, Danecek et al., 2011) and GATK toolkit (VariantFiltration, DePristo et al., 2011) were used to filter the raw variants for strand bias, end distance bias, base quality bias, SNPs around gaps, low coverage and erroneously high coverage. Variants were combined using GATK's CombineVariants (keeping any unfiltered). High quality variants not filtered were annotated using SnpEff (Cingolani et al., 2012).

Three approaches were used to detect structural variants. First, BreakDancer (Chen et al., 2009) was used to predict deletions, insertions, inversions, and translocations using deviations in the separation or orientation of mapped read pairs. Pindel (Ye et al., 2009) was used to infer deletions, short insertions, long insertions, inversions, tandem duplications, and breakpoints using a split-read approach (the output of BreakDancer was also fed to Pindel to improve its output). Finally, Control-FREEC (Boeva et al., 2011) was used to detect copy number variants (CNVs). Control-FREEC finds CNVs using depthof-coverage (normalized by GC-content). Regions of low mappability were excluded by supplying Control-FREEC with mappability tracks generated by gem-mappability (GEM library, Marco-Sola et al., 2012).

\section{Fitness assays in other environments}

In addition to M9-serine, we assayed fitness of PA2449 mutants in three additional media. We replaced L-serine with other carbon sources, to test whether fitness gains were specific to adaptation to growth on L-serine. This included M9-glycine: $4.01 \mathrm{~g} / 1 \mathrm{~L}$-glycine; M9-glucose: $12.5 \mathrm{~g} / 1 \alpha$ - $D$-glucose; and M9KB: $10 \mathrm{~g} / 1$ glycerol, $10 \mathrm{~g} / 1$ proteose peptone no. 3; each with $10.5 \mathrm{~g} / 1 \mathrm{M} 9$ Broth, and $1 \mathrm{ml}$ of 1 $\mathrm{M} \mathrm{MgSO}_{4}$. Competition experiments were performed as described in the main text. 


\section{References}

Chen, K., J. W. Wallis, M. D. McLellan, D. E. Larson, J. M. Kalicki, C. S. Pohl,S. D. McGrath, M. C. Wendl, Q. Zhang, D. P. Locke, et al. 2009. BreakDancer: an algorithm for high-resolution mapping of genomic structural variation. Nature Methods 6:677-681.

Cingolani, P., A. Platts, M. Coon, T. Nguyen, L. Wang, S. J. Land, X. Lu, D. M. Ruden, et al. 2012. A program for annotating and predicting the effects of single nucleotide polymorphisms, SnpEff: SNPs in the genome of Drosophila melanogaster strain w1118; iso-2; iso-3. Fly 6:80-92.

Danecek, P., A. Auton, G. Abecasis, C. A. Albers, E. Banks, M. A. DePristo, R. E. Handsaker, G. Lunter, G. T. Marth, S. T. Sherry, et al. 2011. The variant call format and VCFtools. Bioinformatics 27:2156-2158.

DePristo, M. A., E. Banks, R. Poplin, K. V. Garimella, J. R. Maguire, C. Hartl,A. A. Philippakis, G. del Angel, M. A. Rivas, M. Hanna, et al. 2011. A frame-work for variation discovery and genotyping using next-generation DNA sequencing data. Nature Genetics 43:491-498.

Li, H., B. Handsaker, A. Wysoker, T. Fennell, J. Ruan, N. Homer, G. Marth,G. Abecasis, R. Durbin, et al. 2009. The sequence alignment/map format and SAMtools. Bioinformatics 25:2078-2079.

Marco-Sola, S., M. Sammeth, R. Guigó, and P. Ribeca. 2012. The GEM map-per: fast, accurate and versatile alignment by filtration. Nature Methods 9:1185-1188.

Patel, R. K., and M. Jain. 2012. NGS QC Toolkit: a toolkit for quality control of next generation sequencing data. PLoS One 7:e30619.

Ye, K., M. H. Schulz, Q. Long, R. Apweiler, and Z. Ning. 2009. Pindel: a pattern growth approach to detect break points of large deletions and medium sized insertions from paired-end short reads. Bioinformatics 25:2865-2871. 


\section{Supplementary tables and figure captions}

Table S1: Mutations in selection lineages (order does not imply order of substitution). Bold text indicates putative loss of function mutations (e.g. stop codons, frame-shifts, or indels in key functional domains). Mutations in intergenic regions are abbreviated as 'intergenic'. '+' and '-' indicate insertions and deletions of listed amino acids starting at given position.

\begin{tabular}{|c|c|c|c|c|c|c|c|c|}
\hline & Gene 1 & Gene 2 & Gene 3 & Gene 4 & Mutation 1 & Mutation 2 & Mutation 3 & Mutation 4 \\
\hline \multicolumn{9}{|c|}{ PA2449 (enhancer binding protein involved in regulation of L-glycine/L-serine metabolism) } \\
\hline \multirow[t]{10}{*}{$\mathrm{S} 536 \mathrm{~F} / \Delta \mathrm{PilQ}$} & PA2449 & & & & L143P & & & \\
\hline & PA2449 & & & & R326Q & & & \\
\hline & PA2449 & & & & F428L & & & \\
\hline & PA2449 & PA1046 & & & L154V & silent & & \\
\hline & PA2449 & PA1051 & & & L191V & frame-shift & & \\
\hline & PA2449 & PA1269 & & & A94V & stop gained & & \\
\hline & PA2449 & PA2122 & & & G185C & H81Y & & \\
\hline & PA2449 & morA & & & P288L & M1035T & & \\
\hline & PA2449 & PA2438 & intergenic & & $\mathrm{R} 77 \mathrm{C}$ & +TI15 & intergenic & \\
\hline & PA2449 & PA2449 & bauB & & S308P & A89D & $\mathrm{T} 21 \mathrm{I}$ & \\
\hline \multirow[t]{9}{*}{$+\mathrm{P} 518$} & PA2449 & pilI & & & E180G & frame-shift & & \\
\hline & PA2449 & PA1269 & & & V327L & $\mathrm{A} 55 \mathrm{~T}$ & & \\
\hline & PA2449 & pilR & & & H4Q & T364P & & \\
\hline & PA2449 & pilQ & & & L293Q & stop gained & & \\
\hline & PA2449 & pilC & intergenic & & P288L & frame-shift & intergenic & \\
\hline & PA2449 & pilW & intergenic & & P288L & frame-shift & intergenic & \\
\hline & PA2449 & pilW & intergenic & & S308P & C93R & intergenic & \\
\hline & PA2449 & pilY1 & intergenic & & S308P & frame-shift & intergenic & \\
\hline & PA2449 & pilR & $\operatorname{gacS}$ & intergenic & $\mathrm{R} 77 \mathrm{C}$ & VPPLRE3035 & frame-shift & intergenic \\
\hline \multicolumn{9}{|c|}{ Protease mutants $(c l p A, f t s H, h f l K)$} \\
\hline \multirow[t]{6}{*}{$\overline{\mathrm{S} 536 \mathrm{~F} / \Delta \mathrm{PilQ}}$} & $\operatorname{clp} A$ & $c z c S$ & & & frame-shift & $\mathrm{T} 175 \mathrm{P}$ & & \\
\hline & $c l p A$ & $h f l K$ & & & frame-shift & stop gained & & \\
\hline & $f t s H$ & $g b d R$ & & & R69C & C310R & & \\
\hline & $f t s H$ & $\operatorname{cbr} A$ & & & L81P & F71L & & \\
\hline & $h f l K$ & $\operatorname{cbr} A$ & & & frame-shift & LA324- & & \\
\hline & $h f l K$ & PA4631 & & & frame-shift & F143L & & \\
\hline \multirow[t]{8}{*}{$+\mathrm{P} 518$} & $\operatorname{clp} A$ & & & & frame-shift & & & \\
\hline & $\operatorname{clp} A$ & & & & frame-shift & & & \\
\hline & $\operatorname{clp} A$ & PA0020 & & & GREH195- & frame-shift & & \\
\hline & $\operatorname{clp} A$ & pilB & & & frame-shift & frame-shift & & \\
\hline & $c l p A$ & pilE & & & stop gained & frame-shift & & \\
\hline & $\operatorname{clp} A$ & $\operatorname{alg} R$ & & & frame-shift & R235W & & \\
\hline & $\operatorname{clp} A$ & PA1365 & pilM & & frame-shift & NT794T & frame-shift & \\
\hline & $\operatorname{clp} A$ & PA2063 & pilB & & stop gained & V265I & H421R & \\
\hline \multicolumn{9}{|c|}{ PA2449 and protease ( $c l p A, f t s H, h f l C)$ double mutants } \\
\hline \multirow[t]{5}{*}{ 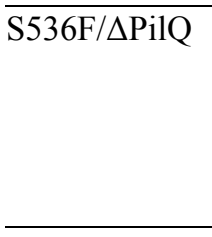 } & PA2449 & $\operatorname{clp} A$ & & & I184S & frame-shift & & \\
\hline & PA2449 & $\operatorname{clp} A$ & & & S308P & V223G & & \\
\hline & PA2449 & $h f l C$ & & & K314M & stop gained & & \\
\hline & PA2449 & $f t s H$ & hutC & & R76L & VFMF115V & $\mathrm{I} 34 \mathrm{~T}$ & \\
\hline & PA2449 & hflC & fruR & PA0340 & G185S & frame-shift & R57P & L88V \\
\hline \multicolumn{9}{|l|}{ Others } \\
\hline $\mathrm{S} 536 \mathrm{~F} / \Delta \mathrm{PilQ}$ & PA2897 & $\operatorname{cbr} A$ & & & V109G & $\mathrm{A} 408 \mathrm{~T}$ & & \\
\hline +P518 & pilB & $c b r B$ & & & I129T & S216P & & \\
\hline
\end{tabular}


Table S2: Description of genes mutated during long-term selection on L-serine.

\begin{tabular}{|c|c|c|c|}
\hline Locus ID & Gene & Function & Relation to RpoN or L-serine metabolism? \\
\hline PA0020 & & hypothetical protein & \\
\hline PA0131 & $\operatorname{bau} B$ & beta-alanine catabolism & \\
\hline PA0340 & & hypothetical protein (putative permease) & \\
\hline PA0410 & pilI & twitching motility protein & Part of an RpoN-regulated network \\
\hline PA0928 & $\operatorname{gac} S$ & sensor/response regulator hybrid & RpoN represses GacA \\
\hline PA1046 & & hypothetical protein (possible beta galactosidase) & \\
\hline PA1051 & & putative gluconate symporter and related permeases & \\
\hline PA1269 & & transcriptional regulator & GntR regulator family \\
\hline PA1365 & & probable siderophore receptor & \\
\hline PA2063 & & hypothetical protein & \\
\hline PA2122 & & hypothetical protein & \\
\hline PA2438 & & hypothetical protein $47 \%$ similarity with $\mathrm{HflC}$ & \\
\hline PA2449 & & probable enhancer binding protein & RpoN bEBP \\
\hline PA2524 & $c z c S$ & phosphorelay sensor kinase activity & \\
\hline PA2620 & $\operatorname{clp} A$ & ATP-binding protease component & Protease component \\
\hline PA2897 & & transcriptional regulator with aminotransferase domain & $\begin{array}{l}\text { Amino acid transport and metabolism, } \\
\text { upstream of } \operatorname{alg} U \text {, GntR regulator family }\end{array}$ \\
\hline PA3563 & fruR & fructose transport system repressor & Carbon utilization modulator \\
\hline PA4526 & pilB & type 4 fimbrial biogenesis protein & Part of an RpoN-regulated network \\
\hline PA4527 & pilC & type 4 fimbrial biogenesis protein & Part of an RpoN-regulated network \\
\hline PA4547 & pilR & two-component response regulator & RpoN bEBP \\
\hline PA4552 & pilW & type 4 fimbrial biogenesis protein & Part of an RpoN-regulated network \\
\hline PA4554 & pilY1 & type 4 fimbrial biogenesis protein & Part of an RpoN-regulated network \\
\hline PA4556 & pile & type 4 fimbrial biogenesis protein & Part of an RpoN-regulated network \\
\hline PA4601 & morA & motility regulator & Upstream of $g l y A 3$ \\
\hline PA4631 & & nucleoside-diphosphate-sugar epimerase & \\
\hline PA4725 & $\operatorname{cbr} A$ & two-component sensor (histidine kinase) & Part of two-component RpoN bEBP \\
\hline PA4726 & $\operatorname{cbr} B$ & two-component response regulator & Part of two-component RpoN bEBP \\
\hline PA4751 & $f t s H$ & ATP-dependent zinc protease & Protease component \\
\hline PA4941 & $h f l C$ & protease subunit & Protease component \\
\hline PA4942 & $h f l K$ & protease subunit & Protease component \\
\hline PA5040 & pilQ & $\begin{array}{l}\text { type } 4 \text { fimbrial biogenesis outer membrane protein } \\
\text { precursor }\end{array}$ & Part of an RpoN-regulated network \\
\hline PA5044 & pilM & type 4 fimbrial biogenesis protein & Part of an RpoN-regulated network \\
\hline PA5105 & hutC & histidine utilization repressor & GntR regulator family \\
\hline PA5261 & $\operatorname{alg} R$ & alginate biosynthesis regulatory protein & algC probably RpoN transcribed \\
\hline PA5380 & $g b d R$ & glycine betaine- and dimethylglycine-responsive regulator & $\begin{array}{l}\text { Betaine is a byproduct of L-serine } \\
\text { catabolism via pss } A\end{array}$ \\
\hline
\end{tabular}

$\mathrm{bEBP}$ - bacterial enhancer binding protein 
Table S3: PA2449 mutations fixed in single mutation selection experiment.

\begin{tabular}{lll}
\hline & Gene & Mutation \\
\hline S536F & PA2449 & R77C \\
S536F & PA2449 & R77C \\
S536F & PA2449 & L143V \\
S536F & PA2449 & P181L \\
S536F & PA2449 & P181S \\
S536F & PA2449 & R183C \\
S536F & PA2449 & A189V \\
S536F & PA2449 & H193D \\
S536F & PA2449 & P263L \\
S536F & PA2449 & P263L \\
S536F & PA2449 & S308P \\
+ P518 & PA2449 & G22D \\
+ P518 & PA2449 & I184V \\
\hline
\end{tabular}

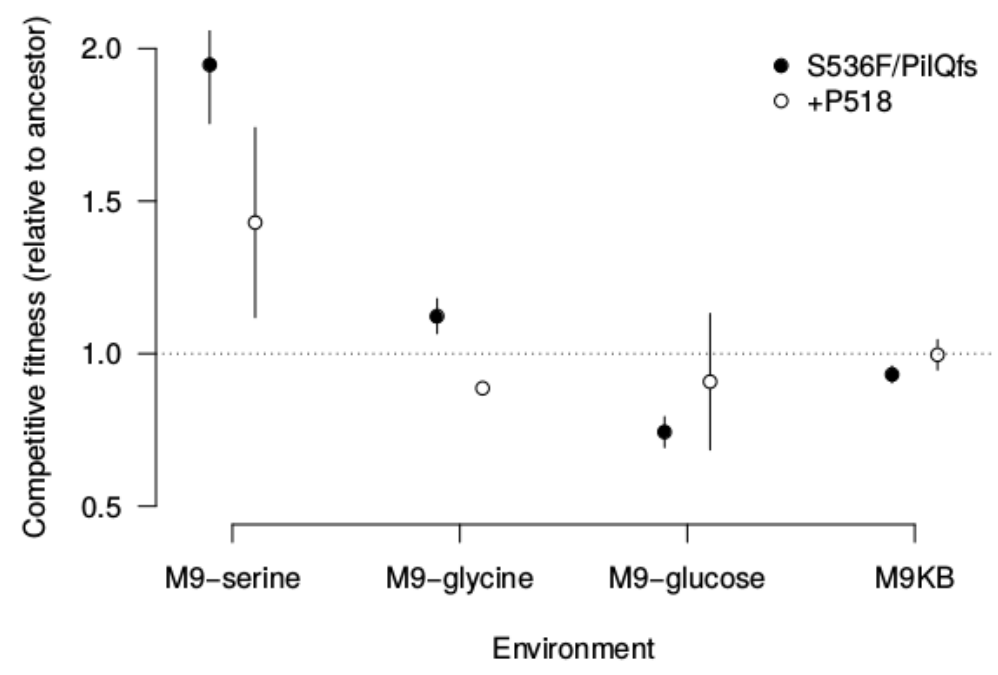

Figure S1: Fitness of PA2449 mutants assayed in M9-serine, M9-glycine, M9-glucose, and M9KB. 


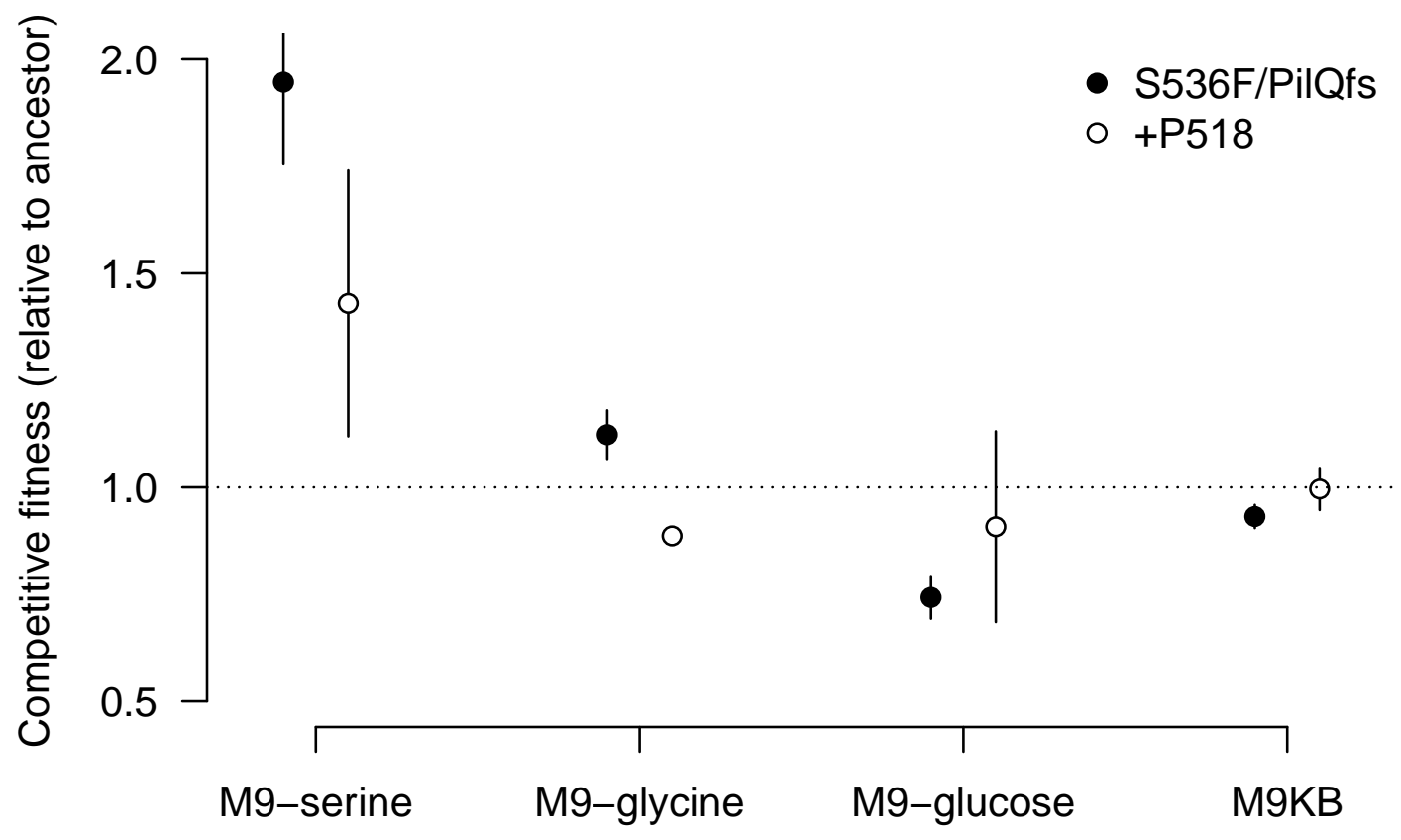

Environment 